\title{
Utilization of network graph connectivity to evaluate complications of inpatient medical and surgical populations as a method to prioritize quality improvement efforts.
}

Bear-Pfaffendorf, Kattie

\section{Abstract}

Network graphs can provide a quantitative framework for identifying complications with significant volumes and strong relationships to other complications, as a method for prioritization of quality improvement work. Here we examine the application of network graphing techniques to acute care inpatient complications on acute care medicalsurgical units of a quaternary care center. The 3M PPC software identified 66 complications among 106 unique patients with two or more complications during an inpatient hospital stay. The network graph highlighted renal failure without dialysis and septicemia and severe infections as highly connected complications in this population.

\section{Background}

Since the inception of the Triple Aim, healthcare has endeavored to measure quality of care (Institute of Medicine, 2000). Complications during inpatient acute care are one aspect of quality of care (Leape, 1994). Acute care hospitals are faced with numerous types of complications including hospital-associated infections, medication errors, and falls (Centers for Medicare \& Medicaid Services, 2016). The plethora of complications to be addressed by hospitals poses an operational challenge in the face of competing priorities.

The evolving field of data visualization continues to offer new approaches to understanding data. Network graphing analysis has been applied to numerous data sets (Viegas \& Donath, 2004). Network graphing techniques allow a better way to understand and appreciate complicated relationships (Dekker \& Colbert, 2004).

Network graphs can provide a quantitative framework for identifying complications with significant volumes and strong relationships to other complications, as an indicator of severity, as a method for prioritization of quality improvement work. Here we examine the application of network graphing techniques to acute care inpatient complications on acute care medical-surgical units of a quaternary care center. 


\section{Methods}

Data was retrospectively analyzed for patients discharged from medical-surgical units at Abbott Northwestern Hospital (Minneapolis, Minnesota) between January and October 2015. Patient level data from the electronic medical record is stored in an electronic data warehouse (EDW). We applied 3M Potentially Preventable Complications software (PCC) (3M, St. Paul, Minnesota) to this data to identify complications and accessed the results through a QlikView dashboard (Qlik, Radnor, Pennsylvania) had two or more complications during a single inpatient stay were used to develop nodes and edge weights. We used Gephi (Paris, France) software to build the network graph (Gephi, 2016)

Each node represents an individual complication and is color coded by PPC complication group. Each edge, or line, represents at least one patient that had both complications; thicker edges represent a more patients with both complications. The arrowheads are an artifact of the software and should be disregarded. In figure 1 each node is labeled with the number corresponding to the PPC description in table 1. 
bioRxiv preprint doi: https://doi.org/10.1101/099150; this version posted January 92017 . The copyright holder for this preprint (which was not

\section{Data}

106 unique patients were found to have two or more complications. The 3M PPC software identified 66 complications, 39 of which occurred in pairs. As illustrated in Table 1, each complication involves a varying number of patients with differing partner complications. There were no pairs of complications that always occurred together.

\section{Figure 1. Network graph of complications in medical surgical patients}

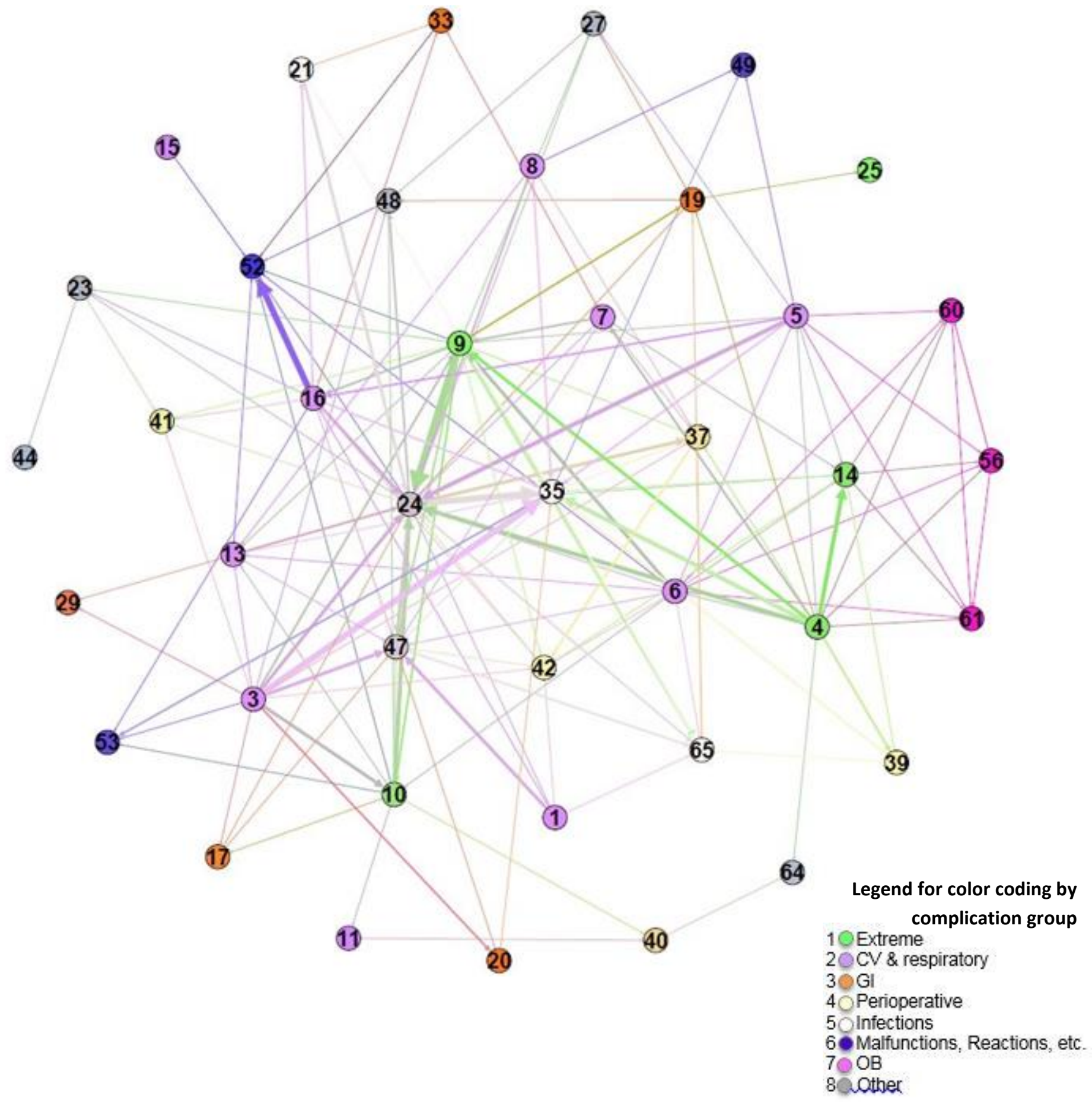




\section{Table 1. Complications}

3M PPC Category

\section{Number of \\ patients Edges w/ PPC}

1: Stroke \& Intracranial Hemorrhage

3: Acute Pulmonary Edema and Respiratory Failure without Ventilation

$3 \quad 7$

4: Acute Pulmonary Edema and Respiratory Failure with Ventilation 18

5: Pneumonia \& Other Lung Infections

6: Aspiration Pneumonia

7: Pulmonary Embolism

8: Other Pulmonary Complications

$14 \quad 27$

9: Shock

10: Congestive Heart Failure

$9 \quad 17$

$4 \quad 16$

11: Acute Myocardial Infarction

$8 \quad 9$

13: Other Cardiac Complications

14: Ventricular Fibrillation/Cardiac Arrest

15: Peripheral Vascular Complications Except Venous Thrombosis

16: Venous Thrombosis

17: Major Gastrointestinal Complications without Transfusion or Significant Bleeding

19: Major Liver Complications

20: Other Gastrointestinal Complications without Transfusion or Significant Bleeding

21: Clostridium Difficile Colitis

23: GU Complications Except UTI

24: Renal Failure without Dialysis

25: Renal Failure with Dialysis

27: Post-Hemorrhagic \& Other Acute Anemia with Transfusion

29: Poisonings except from anesthesia

33: Cellulitis

35: Septicemia \& Severe Infections

37: Post-Operative Infection \& Deep Wound Disruption Without Procedure

39: Reopening Surgical Site

40: Post-Operative Hemorrhage \& Hematoma without Hemorrhage Control Procedure or I\&D Proc

41: Post-Operative Hemorrhage \& Hematoma with Hemorrhage Control Procedure or I\&D Proc

42: Accidental Puncture/Laceration During Invasive Procedure

44: Other Surgical Complication - Mod

47: Encephalopathy

48: Other Complications of Medical Care

49: Iatrogenic Pneumothrax

52: Inflammation \& Other Complications of Devices, Implants or Grafts Except Vascular Infection

53: Infection, Inflammation \& Clotting Complications of Peripheral Vascular Catheters \& Infusions

56: Obstetrical Hemorrhage with Transfusion

64: Other In-Hospital Adverse Events

65: Urinary Tract Infection

3

8

1
4

4

6

1

13

13
2

4

4

2

7

2

34

1

3

3
2
3

3

3

25

7

4

4

2

2

25

$6 \quad 10$

$1 \quad 1$

$12 \quad 22$

$5 \quad 7$

23

$10 \quad 14$

35

16

22

$8 \quad 14$




\section{Results}

The edge between complication 16: Venous Thrombosis and complication 52: Inflammation \& Other Complications of Devices, Implants or Grafts except Vascular Infection, is the strongest edge in the network with a strength of 17. Complication 25: renal failure without dialysis is highly related to other complications, with 60 edges among the 34 patients. Complication 35: Septicemia and Severe infections is also highly connected. The connectivity of complication 35: Septicemia and Severe infections supports the clinical knowledge; patients with sepsis are complex and at risk for additional complications (Society of Critical Care Medicine, 2016).

\section{Discussion}

The inherent challenges of using administrative data in 3M PPC software analysis are well documented in quality improvement literature (Lawthers, 2000) (Geraci, 1999). Clinical experts offer their critiques of the 3M PPC software methodology itself and some have a strong basis (Hughes MD \& al, 2006). To date, relevant literature has not coalesced around a single definitive source of definitions for any complication. The use of the 3M PPC software provides a consistent application of the methodology across cases.

\section{Conclusion}

Use of network graphs to identify complications with high connectivity or centrality may provide a useful framework for identification and prioritization of high impact quality improvement efforts. Being able to target organizational quality and process improvement efforts on connected complications may reduce overall complications at a higher rate than efforts focused on improving complications with low connectivity. 


\section{References}

3M . (2013, 10 1). 3M Potentially Preventable Complications Definations Manual . Retrieved 2015, from ASAP PPC.qvw:

https://prodqv.allina.com/QvAJAXZfc/AccessPoint.aspx?open=\&id=PRODQVS\%7COpe n\%20Documents\%2FPreventables\%2FPPC.qvw\&client=Plugin

Centers for Medicare \& Medicaid Services. (2016). Welcome to the Partnership for Patients.

Retrieved from CMS.gov: https://partnershipforpatients.cms.gov/

Dekker, A. H., \& Colbert, B. D. (2004). Network Robustness and Graph Topology. Canberra, Australia: Defence Science and Technology Organisation (DSTO).

Dimick MD, J. B., \& al, e. (2004). Hospital Costs Associated with Surgical Complications: A Report from the Private-sector National Surgical Quality Improvement Program. the American College of Surgeons, 531-537.

Gephi. (2016). Gephi - The Open Graph Vix Platform. Retrieved from https://gephi.org/

Geraci, J. M. (1999). The Assocciation of Quality of Care and Occurence of In-Hospital, Treatment-Related Complications. Medical Care, 140-149.

Hughes MD, J. S., \& al, e. (2006). Identifying Potentially Preventable Complications Using a Present on Admission Indicator. Health Care Financing Review, 63-82.

Institute of Medicine. (2000). To Err is Human; Committee on Quality of Health Care in America. Washington, DC.: The National Academies Press.

Lawthers, A. G. (2000). Identificaiton of In-Hospital Complications From Claims Data: Is It Valid? Medical Care, 785-795.

Leape, L. (1994). Error in Medicine. JAMA, 1851-1857.

Munzner, T. (2014). Visualization Analysis and Design. A K Peters.

Society of Critical Care Medicine. (2016). Surviving Sepsis Campagin. Retrieved from http://www.survivingsepsis.org/Pages/default.aspx

Viegas, F. B., \& Donath, J. (2004). Social Network Visualization: Can We Go Beyond the Graph? Cambridge, MA: MIT Media Laboratory. 